\title{
Perfil morfofuncional de pacientes com retinopatia diabética sem baixa acuidade visual severa em hospital público de referência em diabetes no Brasil
}

\author{
Morpho-functional profile of patients with diabetic \\ retinopathy without severe loss of visual acuity in a public \\ hospital of reference in diabetes in Brazil
}

Alípio de Sousa Neto ${ }^{1}$, Élida Maria de Araújo Pereira², Regina Cândido Ribeiro dos Santos³ ${ }^{3}$ Procópio Miguel dos Santos ${ }^{4}$

\section{ResUMO}

Objetivo: Demonstrar o perfil morfofuncional da avaliação de pacientes com retinopatia diabética sem baixa acuidade visual severa em hospital público de referência em endocrinologia, determinando nesta amostra, a relação entre o tempo de diabetes, idade e acuidade visual com a espessura retiniana medidas pela tomografia de coerência óptica (OCT) e retinografia (RET). Métodos: Foi realizado estudo prospectivo, linear, em corte transversal de 61 pacientes consecutivos com retinopatia diabética registrados no Hospital Regional de Taguatinga (HRT), e encaminhados pelos serviços de oftalmologia e endocrinologia. Pacientes foram submetidos à avaliação oftalmológica completa, incluindo história clínica, aferição da acuidade visual com correção e com buraco estenopeico. 109 olhos de 55 pacientes foram então submetidos à OCT e RET para avaliação da presença ou ausência de edema macular pela RET e da avaliação quantitativa (medidas da espessura retiniana das 9 regiões classificadas pelo Early Tratment Diabetic Retinophaty Study - ETDRS) e da avaliação qualitativa (presença ou ausência de edema retiniano pela fenda central, número 1). Resultados: $\mathrm{O}$ tempo médio de diabetes entre os sujeitos da pesquisa foi de 12 anos, com idade variando de 23 a 86 anos; $51 \%$ eram do gênero feminino, e $49 \%$ do masculino. A avaliação da acuidade visual melhorou em 47\% (51/ 109) dos pacientes com buraco estenopeico. Somente $22 \%(24 / 109)$ dos olhos apresentavam acuidade visual corrigida de 20/20 com correção e sem edema macular diabético (EMD). Em 83\% (91/109) dos casos havia concordância e em 17\% (18/109 olhos) havia discordância em relação à presença/ausência de EMD no perfil da avaliação morfológica realizada pela OCT e pela RET nos olhos do grupo amostral. Conclusão: Não houve correlação estatística significante entre a espessura retiniana e o tempo de diabetes. $O$ grupo da oftalmologia teve 5 vezes mais chance de apresentar EMD do que o grupo da endocrinologia.

Descritores: Retinopatia diabética; Tomografia de coerência óptica; Edema macular; Técnicas de diagnóstico oftalmológico/utilização; Retina/radiografia; Acuidade visual

\footnotetext{
${ }^{1}$ Pos-doctoral Research Fellowship Schepens Eye Research Institute - Harvard Medical School; Doutor, Chefe do Serviço de Retina e Vítreo do Instituto de Cirurgia Ocular de Brasília - ICOB - Brasília (DF), Brasil;

${ }^{2}$ Chefe do Serviço de Lentes de Contato do Instituto de Cirurgia Ocular de Brasília - ICOB - Brasília (DF), Brasil.

${ }^{3}$ Doutora, Professora da pós-graduação da Faculdade de Ciências da Saúde da Universidade de Brasília - UnB - Brasília (DF);

Professora da Faculdade de Medicina do Distrito Federal - ESCS/FEPECS - Brasília (DF), Brasil;

${ }^{4}$ Doutor, Professor da pós-graduação da Faculdade de Ciências da Saúde da Universidade de Brasília - UnB - Brasília (DF), Brasil; Professor da Faculdade de Medicina do Distrito Federal - ESCS/FEPECS - Brasília (DF), Brasil.

Trabalho realizado no Instituto de Cirurgia Ocular de Brasília (ICOB) e Núcleo de Alta Complexidade em Oftalmologia (NACO), Brasília (DF), Brasil. Este trabalho será parte da tese de doutoramento do primeiro autor (ASN) - Brasília (DF), Brasil.
}

Recebido para publicação em: 1/10/2008 - Aceito para publicação em 5/11/2009 


\section{INTRODUÇÃO}

$\mathbf{V}$ árias doenças oculares são diretamente associadas à alteração da espessura retiniana, tipica mente causada por edema ou atrofia. O edema macular diabético (EMD), por exemplo, é considerado a causa mais frequente de perda visual em casos de retinopatia diabética não proliferativa. $\mathrm{O}$ edema consiste no acúmulo de fluido e no consequente aumento da espessura da retina, gerado pela permeabilidade vascular aumentada, que leva ao vazamento de fluido e plasma, como lipoproteínas, dentro da retina.

A melhor observação do edema se faz por meio de exames estereoscópicos (biomicroscopia ou retinografia em estéreo). Quando o espessamento acomete ou ameaça o centro da mácula, há alto risco de perda da visão. A retinografia em estéreo (RET) é considerada a técnica padrão para diagnóstico do edema macular e forma a base do diagnóstico para o tratamento realizado com o laser, segundo o Early Treatment Diabetic Retinopathy Study (ETDRS) ${ }^{(4)}$, que utiliza como critério de tratamento a área e o espessamento regional.

Nos últimos anos, porém, tecnologias como a tomografia de coerência óptica (OCT) e o analisador de espessura retiniana (RTA) foram criados para possibilitar a quantificação objetiva do edema macular, bem como para documentar e acompanhar o respectivo quadro com alta precisão e boa reprodutibilidade ${ }^{(2,3)}$.

A RET separa,fotograficamente, os tecidos do olho, facilitando a interpretação que pode ser feita com o auxílio de lente especial, tanto na folha de contato ou negativos, como por um visor especial, em frente à tela do computador.A OCT realiza cortes tomográficos em tecidos biológicos com alta resolução, em escala microscópica, medindo o tempo de demora do eco e a intensidade da luz refletida.

O conhecimento do perfil das alterações morfológicas dos olhos com retinopatia diabética, com visão central boa ou moderadamente afetada, evidenciado pelos dois métodos, poderá ter ampla utilização em projetos de saúde ocular na rede pública brasileira. Pretende-se ainda traçar o perfil visual por metodologia de uso fácil (buraco esteropeico) que dispensa equipamentos específicos, podendo ser aplicada por agentes comunitários de saúde em projetos de saúde coletiva. Ao final da pesquisa, foi feita a comparação do perfil de apresentação dos dois métodos, para se traçar o perfil morfofuncional do grupo de pacientes envolvidos no estudo. A utilização dessas técnicas no diagnóstico da retinopatia diabética pode possibilitar o tratamento oftalmológico adequado na rede pública nacional.

\section{Métodos}

\section{Caracterização do estudo}

Trata-se de um estudo prospectivo, linear, analítico-descritivo, em corte transversal, realizado entre 5 de janeiro a 21 de dezembro de 2007.

\section{População e amostra}

O grupo de estudo foi constituído de pacientes cadastrados nos serviços de Endocrinologia e de Oftalmologia do Hospital Regional de Taguatinga (HRT), instituição referência de endocrinologia no Centro-Oeste Brasileiro. Os pacientes foram avaliados inicialmente no serviço de Endocrinologia e referenciados para um parecer oftalmológico, ou primariamente no serviço de Oftalmologia.

Para a realização dos testes específicos (objeto do estudo), foram considerados todos pacientes, após a observação dos critérios de inclusão e de exclusão. Desses seriam excluídos pacientes que apresentassem discordância na interpretação na retinografia colorida (RET).

Critérios de Inclusão: Pacientes com retinopatia diabética, consecutivos, examinados do serviço de Oftalmologia do HRT com o seguinte diagnóstico: diabetes há mais de um ano; adequada visualização do fundo de olho; acuidade visual melhor ou igual a 20/100 e sinais de retinopatia diabética,avaliados pela oftalmoscopia direta ou biomicroscopia de fundus; apresentação de, pelo menos, uma micro-hemorragia e/ou microaneurisma e/ou área retiniana infiltrada por exsudatos duros.

Critérios de Exclusão: Pacientes com: prévia submissão a Laser; catarata de moderada a severa, suficiente para impedir as imagens; submissão a cirurgia intraocular prévia; olhos com hemorragia vítrea; opacidade significante de córnea; outras doenças retinianas que não a retinopatia diabética.

\section{Aspectos éticos}

A investigação foi previamente autorizada pelo Comitê de Ética em Pesquisa (CEP) da Universidade de Brasília e aprovada pelo Comitê de Ética do HRT e pela Comissão Nacional de Ética em Pesquisa (CONEP), por estar de acordo com os padrões éticos exigidos pela Resolução 196/96 do Conselho Nacional de Saúde (CNS), pela Declaração de Helsinki e pelas diretrizes internacionais para as Pesquisas Biomédicas Envolvendo Seres Humanos (CIOMS) (Parecer CONEP n. ${ }^{\circ}$ 79.343).

Todos os pacientes da amostra receberam infor- 


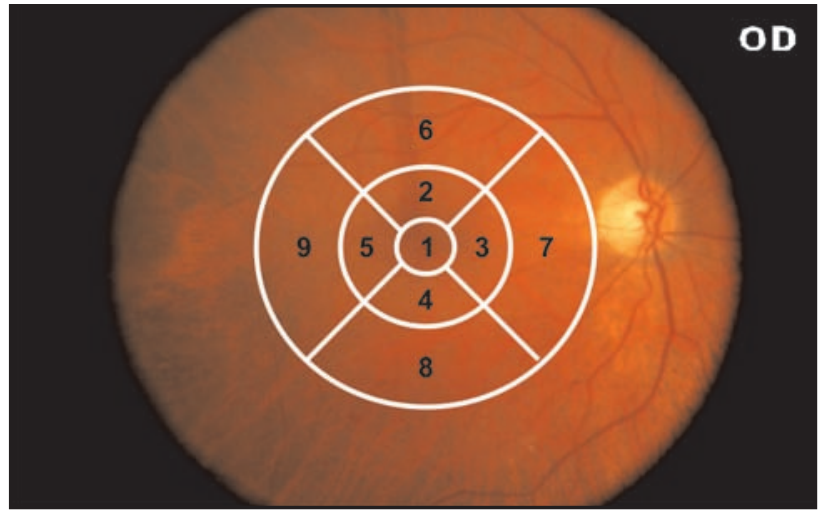

Figura 1: Imagens das regiões obtidas com o retinógrafo

mações completas por escrito e oralmente, de acordo com Termo de Consentimento Livre e Esclarecido. Cada paciente foi arguido quanto a seu entendimento e à suficiência dos esclarecimentos, antes de assinar o referido Termo. Após a assinatura, os pacientes foram conduzidos ao Instituto de Cirurgia Ocular de Brasília (ICOB), dando-se início aos procedimentos de investigação. Eles foram submetidos à anamnese, enfocando-se principalmente o tempo de acometimento de diabetes, a presença de hipertensão arterial sistêmica (HAS) e doenças subjacentes.

Os exames complementares foram realizados em clínicas privadas ICOB e Núcleo de alta complexidade em Oftalmologia (NACO), sem ônus. O autor ofereceu e realizou fotocoagulação a laser em todos os pacientes examinados indicados para esse procedimento. Após o adequado controle das microanormalidades tratadas, foi feito relatório de conclusão do tratamento, incluindo laudo dos exames complementares. Todos os pacientes, incluídos (não tratados e tratados) e excluídos pelos critérios de inclusão/exclusão, receberam laudo conclusivo do exame oftalmológico, dos exames complementares (quando realizados) e indicação para possível acompanhamento e tratamento posterior, como: cirurgia de catarata, cirurgia de vitrectomia posterior.

\section{Exames oftalmológicos e complementares}

Foram realizados exames oftalmológicos completos, com: aferição da pressão intraocular; refração objetiva; aferição da acuidade visual (AV) com correção através de escala de optótipos de Snellen e medida de AV com buraco estenopeico (BE). Optou-se por essa escala por ela representar o padrão utilizado nos consultórios brasileiros. Nessa escala, 20/20 significa 100\% de visão central.
Tabela 1

Medidas de espessura normais por região

\begin{tabular}{ccccc}
\hline Região & Medida Normal & \multicolumn{2}{c}{ IC (95\%) } \\
\hline R1 & $\mathbf{2 1 2}$ & $\mathbf{1 9 2}$ & $-\mathbf{2 3 2}$ \\
R2 & $\mathbf{2 5 5}$ & $\mathbf{2 3 8}$ & $-\mathbf{2 7 2}$ \\
R3 & $\mathbf{2 6 7}$ & $\mathbf{2 5 1}$ & - & $\mathbf{2 8 3}$ \\
R4 & $\mathbf{2 6 0}$ & $\mathbf{2 4 5}$ & - & $\mathbf{2 7 5}$ \\
R5 & $\mathbf{2 5 1}$ & $\mathbf{2 3 8}$ & - & $\mathbf{2 6 4}$ \\
R6 & $\mathbf{2 3 9}$ & $\mathbf{2 2 3}$ & - & $\mathbf{2 5 5}$ \\
R7 & $\mathbf{2 4 6}$ & $\mathbf{2 3 2}$ & $-\mathbf{2 6 0}$ \\
R8 & $\mathbf{2 1 0}$ & $\mathbf{1 9 7}$ & $-\mathbf{2 2 3}$ \\
R9 & $\mathbf{2 1 0}$ & $\mathbf{1 9 6}$ & $-\mathbf{2 2 4}$ \\
\hline
\end{tabular}

Fonte: Chan e Duker ${ }^{(3)}$

Após os procedimentos iniciais, fez-se a dilatação pupilar com Mydriacyl ${ }^{\circledR} 1 \%$, uma gota a cada 15 minutos, 3 vezes. Nos pacientes que não conseguiram boa midríase, aplicou-se uma gota de Fenilefrina ${ }^{\circledR} 10 \%$. Posteriormente à dilatação pupilar, os pacientes foram submetidos pelo autor à oftalmoscopia binocular indireta $\mathrm{e}$ à biomicroscopia de fundo através de lente de condensação de 78 dioptrias positivas. Foram então encaminhados para a realização de OCT e RET.

Os exames de RET e de OCT foram feitos por diferentes examinadores. Os de RET foram avaliados pelo pesquisador e por outro analista.

No período em que permaneceram no ICOB, os pacientes receberam alimentos balanceados, de modo a evitar quadros de hipoglicemia. Ao término dos exames, foram levados de volta ao HRT.

Medida da acuidade visual e com buraco estenopeico

A medida da AV foi realizada por meio de um projetor de optótipos com escala padrão Snellen. Cada paciente foi colocado a seis metros de distância do optótipo, em um ambiente com iluminação adequada. Inicialmente, a medida foi realizada com os óculos; depois, quando a visão não era de 20/20, colocava-se máscara com BE, que era segurada pelo paciente a $1 \mathrm{~cm}$ das pálpebras, por onde ele buscava a melhor visão olhando por um dos dezoito orifícios. Considerou-se que caso houvesse melhora da $\mathrm{AV}$, isso significava que a visão deveria melhorar com a mudança no grau dos óculos.

\section{Exame de retinografia}

$\mathrm{Na}$ retinografia, as imagens foram obtidas com ângulo de 35 graus (equivalente ao campo 2 do ETDRS), feitas com retinógrafo TOPCON 50TRC 50EX-Japão, digitalizado por meio do software MEDLAB $\AA$, com 

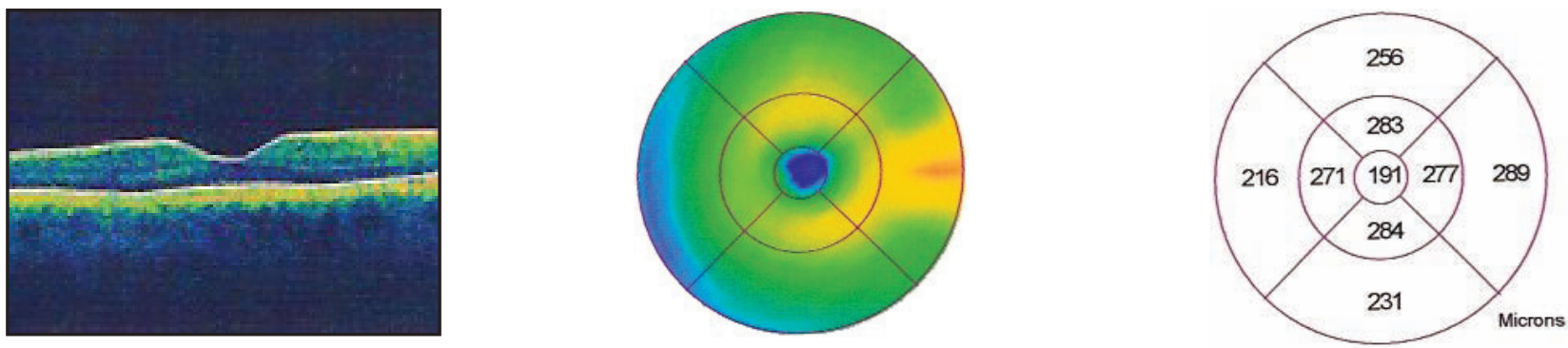

Figura 2: Medidas por área

Tabela 2

Medidas descritivas da idade/anos, tempo de diabetes (anos)

\begin{tabular}{lcccccc}
\hline & N & Mínimo & Máximo & Média & Desvio-padrão & IC (95\%) \\
\hline Idade & 55 & 23 & 86 & 58,30 & 12,92 & $54,96-61,64$ \\
Tempo de diabetes & 55 & 1 & 38 & 12,18 & 8,66 & $9,93-14,44$ \\
\hline
\end{tabular}

câmera digital Nikon D40 ${ }^{\circledR}$ (Japão). As imagens foram apresentadas em PowerPoint (figura 1), com máscara das regiões aplicada eletronicamente sobre a foto, para normatizar a área correspondente, similar à da OCT.

Após a avaliação das fotos com a aplicação de máscaras das nove áreas, correspondentes à grade aplicada pelo Early Treatment Diabetic Retinophaty Study (ETDRS), sem conhecer os resultados uns dos outros, os analistas determinaram a ausência ou presença de edema, sem proceder a sua quantificação.

Considerou-se que edema macular clinicamente significativo de acordo com os critérios do ETDRS é aquele que apresenta um espessamento da retina a $500 \mu \mathrm{m}$ do centro da mácula; exsudatos duros, a 500 $\mu \mathrm{m}$ do centro da mácula, se associado à retina adjacente; áreas de espessamento de um disco de diâmetro ou mais dentro de um disco de diâmetro no centro da mácula. Pela retinografia, foram verificados os olhos com edema, a presença de espessamento ou de exsudatos duros em qualquer das nove regiões mostradas na figura 1. Eram considerados olhos sem edema quando havia ausência completa desses achados.

\section{Exame de tomografia de coerência óptica (OCT)}

Nesses exames, foi utilizado o aparelho OCT Stratus 3000 (Carl Zeiss Medtec, Dublin Califórnia) disponível comercialmente, com relatório de análise versão 4.0.1. Esse modelo contém um interferômetro com escaneamento de baixa coerência, trabalhando com comprimento de onda de $840 \mu \mathrm{m}$ com vídeo-câmera que demonstra a visualização da retina. Cada corte representa 100 A-scans longitudinais. Um conjunto de seis
Tabela 3

\section{Correlação entre a idade do paciente e a espessura da retina}

\begin{tabular}{ccc}
\hline $\begin{array}{c}\text { Espessura da } \\
\text { retina pela OCT }\end{array}$ & $\begin{array}{c}\text { Medida da } \\
\text { correlação de Pearson }\end{array}$ & Valor de p \\
\hline OCT_R1 & $-0,031$ & 0,749 \\
OCT_R2 & $-0,059$ & 0,547 \\
OCT_R3 & $-0,170$ & 0,081 \\
OCT_R4 & $\mathbf{- 0 , 2 7 4}$ & $0,004^{*}$ \\
OCT_R5 & $\mathbf{- 0 , 2 3 3}$ & $0,016^{*}$ \\
OCT_R6 & $\mathbf{- 0 , 2 7 2}$ & $0,005^{*}$ \\
OCT_R7 & $\mathbf{- 0 , 3 4 2}$ & $0,000^{*}$ \\
OCT_R8 & $\mathbf{- 0 , 3 3 7}$ & $0,000^{*}$ \\
OCT_R9 & $\mathbf{- 0 , 3 5 8}$ & $0,000^{*}$ \\
\hline
\end{tabular}

* Estatisticamente significativo valor de $\mathrm{p}<0,05$

scans lineares foi colocado igualmente, com intervalos de $30^{\circ}$, cobrindo $6 \mathrm{~mm}$ do polo posterior centrado na fóvea.

A espessura retiniana foi obtida automaticamente em cada localização e representada graficamente entre os scans com o auxílio do software de análise da OCT. Com base nos valores obtidos, a média da espessura retiniana foi calculada automaticamente nas nove áreas predefinidas pelo ETDRS.

\section{Avaliação quantitativa da espessura retiniana}

$\mathrm{Na}$ avaliação quantitativa da espessura retiniana registraram-se os valores de espessura de cada região, fornecidos pela diferença entre reflectividade da face anterior da retina e da face posterior (epitélio pigmentar da retina) (figura 2). 

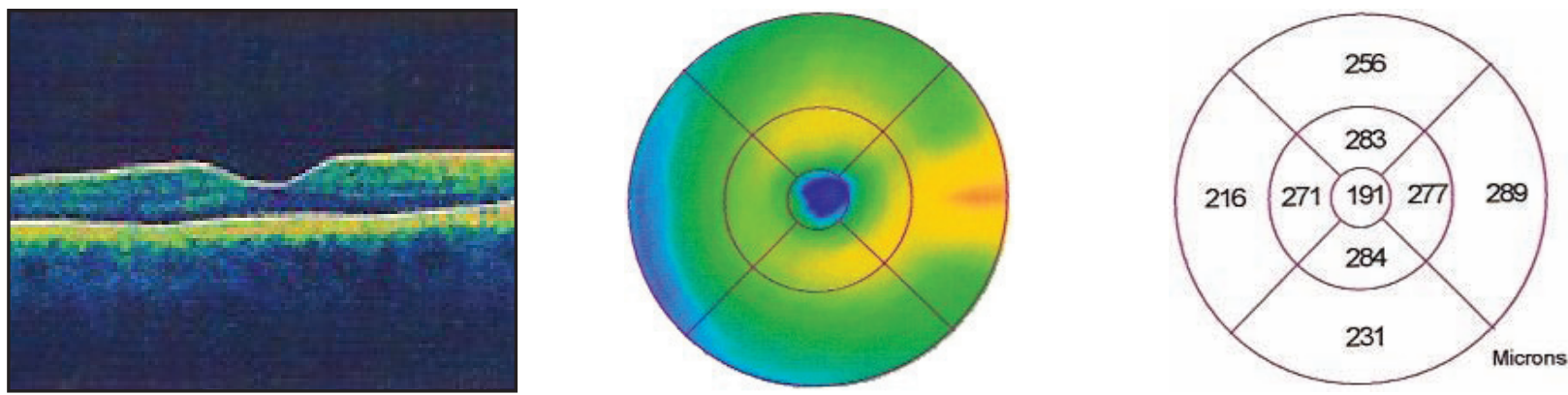

Figura 3 A: - Avaliação correta pelo software do OCT
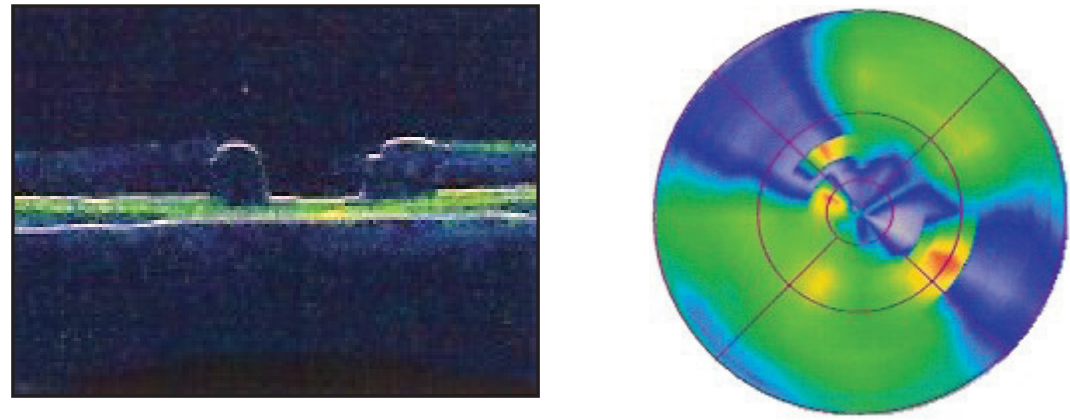

Figura 3 B:- Avaliação incorreta pelo software do OCT

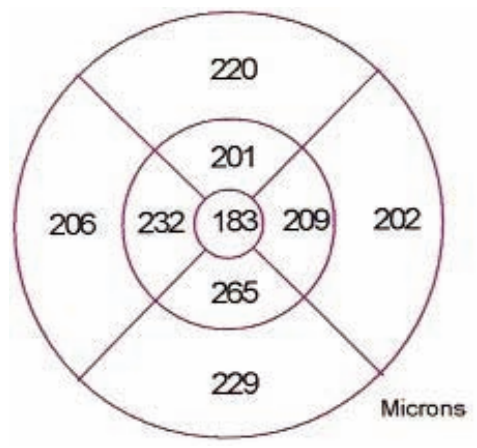

Para verificar a diferença dos valores da OCT entre os pacientes com edema e os sem edema, calculou-se a média dos valores da tomografia nas nove regiões do olho, com um intervalo de confiança de $95 \%$. Foram considerados com edema os olhos com valores acima da média por regiões descritas no estudo de Chan e Duker $^{(3)}$. (tabela 1).

\section{Avaliação qualitativa}

Após a avaliação dos exames, constatou-se que alguns deles não apresentavam leitura correta da espessura pelo software (figuras $3 \mathrm{~A}$ e B), por haver áreas de pouca reflectividade da face anterior da retina. Com isso, fez-se também uma análise qualitativa, avaliando-se, pela fenda, se havia ou não edema macular, sendo os resultados comparados com os dados do software na análise estatística. Na análise qualitativa, foram considerados com edema os tomogramas cuja fenda central (1) apresentasse retificação ou inversão do umbus foveal. Foram impressos laudos das espessuras, similares à grade do ETDRS, e fendas centrais, por meio dos quais pôde ser feita uma avaliação e classificação qualitativa.

\section{Conservação e Tratamento dos Dados}

Os dados foram registrados em fichas clínicas individuais, e os registros foram mantidos aos cuidados do investigador principal, ficando a critério dele a forma e o período em que seriam divulgados.

Inicialmente, foi aplicado o teste de Wilcoxon ${ }^{(2,5)}$ para verificar se a avaliação e o diagnóstico de cada paciente foram os mesmos para os dois pesquisadores.

A hipótese estatística nula teve como princípio avaliar se os resultados verificados com a retinografia foram compatíveis com os apresentados na OCT, o que foi feito por meio da Análise de Variância (ANOVA) ${ }^{(1,5)}$ e o teste de $\mathrm{McNemar}^{(2,5)}$, com relação ao diagnóstico (presença EMD pela RET, e pela OCT)

Em outra comparação, verificou-se se o grupo de pacientes da Oftalmologia e da Endocrinologia era equivalente entre si, quanto à presença de edema macular diabético e à visão. Não foi avaliado o controle metabólico neste estudo, porque a hipótese alternativa fundamentou-se no princípio de que, nesses grupos, haveria diferença de EMD, pelo fato de os pacientes da Oftalmologia estarem em acompanhamento há mais tempo. A comparação também foi verificada pela Análise de Variância (ANOVA) ${ }^{(1,5)}$, pela odds-ratio ${ }^{(5)}$ e pelo Teste Qui-quadrado ${ }^{(1,5)}$, que mostrou a chance de um grupo apresentar edema em relação ao outro. O coeficiente de correlação de Pearson, que mede o grau de associação entre duas variáveis quantitativas, foi utilizado para verificar a associação entre os resultados da OCT e a es- 
Tabela 4

Média de espessura das regiões por grupo de origem e IC (95\%)

\begin{tabular}{lccccc}
\hline & \multicolumn{2}{c}{ Endocrinologia } & \multicolumn{2}{c}{ Oftalmologia } & \\
& Média & IC (95\%) & Média & IC (95\%) & Valor de p \\
\hline OCT_R1 & 191,98 & $184,36-199,60$ & 235,78 & $213,10-258,45$ & $\mathbf{0 , 0 0 1}$ \\
OCT_R2 & 222,63 & $210,35-234,92$ & 261,74 & $238,76-284,73$ & $\mathbf{0 , 0 0 5}$ \\
OCT_R3 & 258,41 & $250,04-266,78$ & 282,79 & $264,72-300,87$ & $\mathbf{0 , 0 2 3}$ \\
OCT_R4 & 258,61 & $248,60-268,63$ & 283,55 & $263,96-303,14$ & $\mathbf{0 , 0 3 4}$ \\
OCT_R5 & 253,73 & $244,85-262,62$ & 278,43 & $264,40-297,46$ & $\mathbf{0 , 0 2 8}$ \\
OCT_R6 & 243,43 & $235,50-251,35$ & 268,28 & $251,98-284,57$ & $\mathbf{0 , 0 1 1}$ \\
OCT_R7 & 237,27 & $230,56-243,98$ & 262,98 & $249,66-276,31$ & $\mathbf{0 , 0 0 1}$ \\
OCT_R8 & 231,94 & $223,99-239,89$ & 256,60 & $241,03-272,17$ & $\mathbf{0 , 0 0 9}$ \\
OCT_R9 & 217,08 & $211,64-222,52$ & 244,07 & $229,16-258,98$ & $\mathbf{0 , 0 0 2}$ \\
\hline
\end{tabular}

Estatisticamente significativo p-valor $<0,05$

Tabela 5

Média de espessura retiniana por regiões (OCT quantitativo). Comparação entre espessuras normais, com edema e sem edema IC (95\%)

\begin{tabular}{|c|c|c|c|c|c|c|c|c|c|c|}
\hline \multirow{3}{*}{$\begin{array}{c}\text { Região } \\
\text { R1 }\end{array}$} & \multirow{3}{*}{$\begin{array}{c}\text { Medida } \\
\text { Normal }\end{array}$} & \multirow{2}{*}{\multicolumn{2}{|c|}{ IC $(95 \%)$}} & \multicolumn{3}{|c|}{ Sem edema n: 91} & \multicolumn{4}{|c|}{ Com edema n: 18} \\
\hline & & & & \multirow{2}{*}{$\begin{array}{l}\text { Média } \\
196,53\end{array}$} & \multicolumn{2}{|c|}{ IC $(95 \%)$} & \multirow{2}{*}{$\begin{array}{l}\text { Média } \\
312,72\end{array}$} & \multicolumn{3}{|c|}{ IC $(95 \%)$} \\
\hline & & 192 & -232 & & 190,83 & $-202,24$ & & 253,58 & & 371,87 \\
\hline $\mathrm{R} 2$ & 255 & 238 & -272 & 225,34 & 216,13 & - 234,55 & 336,00 & 279,99 & - & 392,01 \\
\hline R3 & 267 & 251 & -283 & 259,24 & 252,72 & $-265,76$ & 334,72 & 287,04 & - & 382,40 \\
\hline $\mathrm{R} 4$ & 260 & 245 & - 275 & 259,20 & 251,52 & - 266,89 & 337,67 & 286,07 & - & 389,26 \\
\hline $\mathrm{R} 5$ & 251 & 238 & -264 & 256,61 & 249,37 & $-263,86$ & 320,89 & 267,57 & - & 374,21 \\
\hline R6 & 239 & 223 & -255 & 247,05 & 240,43 & $-253,66$ & 307,78 & 264,35 & - & 351,21 \\
\hline $\mathrm{R} 7$ & 246 & 232 & -260 & 241,77 & 236,20 & $-247,35$ & 300,06 & 266,66 & - & 333,45 \\
\hline $\mathrm{R} 8$ & 210 & 197 & -223 & 235,26 & 228,77 & - 241,76 & 297,22 & 257,02 & - & 337,43 \\
\hline R9 & 210 & 196 & -224 & 221,78 & 216,69 & - 226,88 & 281,61 & 240,62 & - & 322,60 \\
\hline
\end{tabular}

Tabela 6

Comparação da medida normal da espessura da retina com EMD

\begin{tabular}{cccccc}
\hline \multirow{2}{*}{ Região } & \multirow{2}{*}{$\begin{array}{c}\text { Medida } \\
\text { normal }\end{array}$} & \multicolumn{2}{c}{ Sem edema n=91 } & \multicolumn{2}{c}{ Com edema n=18 } \\
\cline { 2 - 5 } & Média & Valor de $\mathbf{p}$ & Média & Valor de p \\
\hline R1 & $\mathbf{2 1 2}$ & 196,53 & $\mathbf{0 , 0 0 0 *}$ & 312,72 & $\mathbf{0 , 0 0 2} *$ \\
R2 & $\mathbf{2 5 5}$ & 225,34 & $\mathbf{0 , 0 0 0} *$ & 336,00 & $\mathbf{0 , 0 0 7} *$ \\
R3 & $\mathbf{2 6 7}$ & 259,24 & $\mathbf{0 , 0 2 0} *$ & 334,72 & $\mathbf{0 , 0 0 8}^{*}$ \\
R4 & $\mathbf{2 6 0}$ & 259,20 & 0,838 & 337,67 & $\mathbf{0 , 0 0 6} *$ \\
R5 & $\mathbf{2 5 1}$ & 256,61 & 0,127 & 320,89 & $\mathbf{0 , 0 1 3}^{*}$ \\
R6 & $\mathbf{2 3 9}$ & 247,05 & $\mathbf{0 , 0 1 8} *$ & 307,78 & $\mathbf{0 , 0 0 4} *$ \\
R7 & $\mathbf{2 4 6}$ & 241,77 & 0,136 & 300,06 & $\mathbf{0 , 0 0 3} *$ \\
R8 & $\mathbf{2 1 0}$ & 235,26 & $\mathbf{0 , 0 0 0} *$ & 297,22 & $\mathbf{0 , 0 0 0} *$ \\
R9 & $\mathbf{2 1 0}$ & 221,78 & $\mathbf{0 , 0 0 0} *$ & 281,61 & $\mathbf{0 , 0 0 2} *$ \\
\hline
\end{tabular}

* Estatisticamente significativo valor de $\mathrm{p}<0,05$ 
pessura da retina. Para a relação entre espessura da retina e a RET, foram utilizados o teste Qui-quadrado e a ANOVA.

Para todos os testes, foram adotados níveis de significância de $5 \%$ (p-valor $<0,05)$. O programa utilizado para a análise dos dados foi $o$ Statistical Package for Social Science (SPSS), versão 13.0.

\section{Resultados}

Durante o período de estudo, foram examinados 61 pacientes com retinopatia diabética, não submetidos à laserterapia, satisfazendo aos critérios de inclusão estabelecidos no protocolo. Todos os pacientes foram submetidos aos exames oftalmológicos completos antes da realização dos exames complementares. Várias razões determinaram a não inclusão de 13 olhos (ambos os olhos de seis pacientes e um olho de um paciente) no grupo amostral: catarata em dez olhos, hemorragia vítrea em dois olhos e discordância na interpretação na RET entre os examinadores em um olho. O grupo amostral se constituiu, portanto, de 55 pacientes, ou seja, de 109 olhos.

Dos 55 pacientes, $28(51 \%)$ eram do gênero feminino, e $27(49 \%$ ), do gênero masculino. A média de idade foi de 58 anos (58 $\pm 12,92$ anos); com variação de 23 a 86 anos.

O tempo médio de diabetes dos pacientes era de 12 anos (12 $\pm 8,88$ anos), variando de 1 a 38 anos de diagnóstico.

A tabela 2 contém os valores descritivos das variáveis, idade, tempo de diabetes e de hipertensão medidas em anos.

\section{Tomografia de Coerência Óptica (OCT)}

\section{OCT x Idade}

Comparando a espessura da retina verificada na OCT em relação à idade dos pacientes, verificou-se, pelo teste de Pearson (tabela 3), que havia uma correlação negativa com significância estatística entre essas duas variáveis nas regiões 4, 5, 6, 7, 8 e 9 do olho.

\section{Tempo de Diabetes x Edema}

$\mathrm{Na}$ análise dos resultados da OCT, analisou-se detidamente a presença ou a ausência de edema nos tomogramas dos pacientes, devido ao software apresentar falhas na leitura das imagens. A partir dessa variável codificada, utilizou-se o ANOVA para verificar se a presença ou não do edema, pela OCT, estaria relacionada com o tempo do diabetes.
Tabela 7

Origem dos pacientes por resultado do OCT

\begin{tabular}{lrrrr}
\cline { 2 - 5 } & \multicolumn{4}{c}{ Resultado OCT } \\
\cline { 2 - 5 } & \multicolumn{3}{c}{ sem edema } & \multicolumn{2}{c}{ com edema } \\
\cline { 2 - 5 } & N & $\%$ & N & $\%$ \\
\hline $\begin{array}{l}\text { Pacientes do ambulatório } \\
\text { de Endocrinologia }\end{array}$ & 50 & 55,5 & - & - \\
$\begin{array}{l}\text { Pacientes do ambulatório } \\
\text { de Oftalmologia }\end{array}$ & 40 & 44,5 & 19 & 32 \\
Total & 90 & 100,0 & 19 & 100,0 \\
\hline
\end{tabular}

Tabela 8

\section{Relação entre o tempo de diabetes e a presença de EMD na retinografia}

\begin{tabular}{cccc}
\hline \multirow{2}{*}{$\begin{array}{c}\text { Região } \\
\text { do olho }\end{array}$} & \multicolumn{2}{c}{$\begin{array}{c}\text { Tempo médio } \\
\text { de diabetes (em anos) }\end{array}$} & Valor de p \\
\cline { 2 - 3 } com edema & sem edema & \\
\hline 1 & 17,6 & 18,0 & 0,819 \\
2 & 17,7 & 17,8 & 0,926 \\
3 & 17,9 & 17,2 & 0,719 \\
4 & 17,9 & 17,3 & 0,751 \\
5 & 17,6 & 17,9 & 0,864 \\
6 & 17,0 & 19,0 & 0,213 \\
7 & 17,5 & 18,3 & 0,643 \\
8 & 17,4 & 18,2 & 0,621 \\
9 & 16,3 & 20,0 & $0,020^{*}$ \\
\hline
\end{tabular}

* Estatisticamente significativo valor de $\mathrm{p}<0,05$

Tabela 9

Teste Qui-quadrado e odds-ratio da retinografia em relação ao grupo de procedência do paciente por olho e região

\begin{tabular}{ccc}
\hline Região do olho & Odds-ratio & Qui-quadrado \\
\hline R1 & 3,4 & $\mathbf{0 , 0 0 6 *}$ \\
R2 & 5,2 & $\mathbf{0 , 0 0 0}^{*}$ \\
R3 & 6,2 & $\mathbf{0 , 0 0 0}^{*}$ \\
R4 & 6,3 & $\mathbf{0 , 0 0 1 *}$ \\
R5 & 4,7 & $\mathbf{0 , 0 0 2}^{*}$ \\
R6 & 4,5 & $\mathbf{0 , 0 0 1}^{*}$ \\
R7 & 5,3 & $\mathbf{0 , 0 0 1 *}$ \\
R8 & 18,5 & $\mathbf{0 , 0 0 0 *}$ \\
R9 & 4,5 & $\mathbf{0 , 0 0 1 *}$ \\
\hline
\end{tabular}

* Estatisticamente significativo valor de $\mathrm{p}<0,05$ 
Tabela 10

Tempo médio de diabetes em relação à presença de EMD

\begin{tabular}{cc}
\hline Presença de EMD & Tempo médio de diabetes (anos) \\
\hline Não & 11,24 \\
Sim & 16,22 \\
Total & 13,73 \\
\hline
\end{tabular}

Tabela 11

Diferenças entre a AV com correção e com buraco estenopeico

\begin{tabular}{lrr}
\hline Relação & N & \% \\
\hline Melhora & 51 & 47 \\
Não melhora & 58 & 53 \\
Piora & 0 & 0 \\
Total & 109 & 100,0 \\
\hline
\end{tabular}

Tabela 12

Melhora da visão por grupo de origem dos pacientes

\begin{tabular}{|c|c|c|c|c|c|c|}
\hline \multirow[t]{2}{*}{$\begin{array}{l}\text { Origem dos } \\
\text { pacientes }\end{array}$} & \multicolumn{2}{|c|}{$\begin{array}{l}\text { melhora } \\
\text { a visão }\end{array}$} & \multicolumn{2}{|c|}{$\begin{array}{l}\text { não melhora } \\
\text { a visão }\end{array}$} & \multicolumn{2}{|c|}{ Total } \\
\hline & $\mathbf{N}$ & $\%$ & $\mathbf{N}$ & $\%$ & $\mathbf{N}$ & $\%$ \\
\hline endo & 23 & 45 & 27 & 47 & 50 & 45 \\
\hline oftalr & 28 & 55 & 31 & 53 & 59 & 55 \\
\hline total & 51 & 100,0 & 58 & 100,0 & 109 & 100,0 \\
\hline
\end{tabular}

Com a aplicação do teste ANOVA, verificou-se que não houve diferença significativa entre a presença de edema e o tempo de diabetes no diagnóstico feito com a OCT.

Pela correlação de Pearson, não se registrou resultado significativo entre a correlação da medida da espessura da retina nas regiões do olho e o tempo de diagnóstico de diabetes.

\section{Análise quantitativa pelo software do equipamento}

Havia 18 olhos (16\%) com edema macular diabético. Foi realizada a comparação entre a média quantitativa da espessura retiniana por região entre os olhos dos pacientes da Endocrinologia e Oftalmologia.

Utilizando os resultados da OCT obtidos com o software para cada um dos grupos (endocrinologia e oftalmologia), obteve-se, pela análise de variância, a relação entre as medidas do programa para cada grupo de pacientes.
Analisando todos os olhos, observou-se que houve diferença significativa entre as medidas da retina pela OCT.Em média, os olhos dos pacientes da oftalmologia apresentaram maior valor na medida da espessura da retina do que os olhos dos pacientes da endocrinologia (tabela 4).

Para verificação das diferenças dos valores da OCT entre os pacientes com edema e os sem edema, determinados pela avaliação qualitativa, calculou-se a média dos valores da tomografia nas nove regiões do olho, com um intervalo de confiança de $95 \%$, comparados à média das medidas normais (Tabela 5).

Em relação ao padrão de normalidade, houve diferença entre a medida da espessura da retina nas regiões 4, 5, 6, 8 e 9 nos olhos estudados. Essas regiões apresentam a retina mais espessa que a medida normal (tabela 6 ).

Os pacientes sem edema tiveram medidas diferentes da considerada normal nas regiões 1,2,3, 6, 8 e 9 . Nas regiões 1, 2 e 3, as medidas da espessura da retina foram menores que a normal, e nas regiões 6,8 e 9, a espessura da retina foi maior que a medida normal.

Nos pacientes com edema, todas as regiões do olho apresentaram medidas da espessura da retina maior que a medida considerada normal.

\section{Análise qualitativa}

Na avaliação da fenda 1 (umbus foveal), houve diferença significativa quanto à presença de edema entre os grupos. Os olhos dos pacientes da oftalmologia tiveram mais chance de apresentar edema do que os pacientes da endocrinologia (valor de $\mathrm{p}<0,005$ ) (tabela 7).

Pela análise qualitativa, nenhum paciente da endocrinologia apresentou edema na OCT, enquanto 19 $(32 \%)$ dos pacientes da oftalmologia apresentaram EMD. A relação entre os grupos e a presença de edema é significativa (valor de $\mathrm{p}=0,001$ ).

\section{Retinografia}

\section{Tempo de Diabetes e Edema Macular}

Na relação entre tempo de diabetes e presença de edema pela RET, verificou-se, pela ANOVA, se houve associação entre o tempo de diabetes dos pacientes com mais de dez anos de diagnóstico e a presença de EMD.

Em média, o tempo de diabetes dos pacientes com EMD foi igual aos daqueles sem EMD avaliado na RET. Apenas a região 9 apresentou significância estatística na associação entre tempo de diabetes e presença de edema (tabela 8 ). 
Tabela 13

Frequência por grupo e melhora da visão com buraco estenopeico

\begin{tabular}{ccccc}
\hline \multicolumn{2}{c}{$\begin{array}{c}\text { Endocrinologia } \\
\text { Melhora }\end{array}$} & $\begin{array}{c}\text { Visão com } \\
\text { Não melhora }\end{array}$ & $\begin{array}{c}\text { Oftalmologia } \\
\text { buraco estenopeico }\end{array}$ & $\begin{array}{cccc}\text { Melhora } \\
\text { Não melhora }\end{array}$ \\
\hline 9 & 18 & $\mathbf{2 0 / 2 0}$ & 2 & 6 \\
4 & 2 & $\mathbf{2 0 / 2 5}$ & 8 & 2 \\
4 & 1 & $\mathbf{2 0 / 3 0}$ & 4 & 8 \\
3 & 4 & $\mathbf{2 0 / 4 0}$ & 5 & 3 \\
1 & 1 & $\mathbf{2 0 / 6 0}$ & 3 & 6 \\
1 & 1 & $\mathbf{2 0 / 8 0}$ & 2 & 2 \\
1 & 0 & $\mathbf{2 0 / 1 0 0}$ & 4 & 4 \\
\hline $\mathbf{2 3}$ & $\mathbf{2 7}$ & TOTAL & $\mathbf{2 8}$ & $\mathbf{3 1}$ \\
\hline
\end{tabular}

Tabela 14

Medidas descritivas do OCT em relação à melhora da visão por olho

\begin{tabular}{|c|c|c|c|c|c|c|c|}
\hline \multirow{3}{*}{$\begin{array}{c}\text { Região } \\
\text { R1 }\end{array}$} & \multicolumn{3}{|c|}{ Não melhora } & \multicolumn{4}{|c|}{ Melhora } \\
\hline & \multirow{2}{*}{$\begin{array}{l}\text { Média } \\
222,50\end{array}$} & \multicolumn{2}{|c|}{ IC (95\%) } & \multirow{2}{*}{$\begin{array}{l}\text { Média } \\
204,80\end{array}$} & \multicolumn{3}{|c|}{ IC (95\%) } \\
\hline & & 201,80 & $-\quad 243,20$ & & 194,85 & - & 214,76 \\
\hline $\mathrm{R} 2$ & 247,71 & 227,13 & $-268,29$ & 237,59 & 221,34 & - & 253,83 \\
\hline $\mathrm{R} 3$ & 276,68 & 260,17 & - 293,20 & 263,49 & 255,10 & - & 271,88 \\
\hline $\mathrm{R} 4$ & 278,38 & 260,71 & - 296,05 & 262,07 & 251,05 & - & 273,09 \\
\hline R5 & 266,52 & 248,94 & - 284,09 & 268,10 & 259,69 & - & 276,51 \\
\hline R6 & 257,85 & 244,03 & - 271,66 & 255,37 & 242,49 & - & 268,24 \\
\hline R7 & 253,26 & 240,94 & - 265,58 & 247,90 & 239,83 & - & 255,97 \\
\hline $\mathrm{R} 8$ & 250,14 & 236,36 & - 263,91 & 237,54 & 226,98 & - & 248,09 \\
\hline R9 & 228,98 & 216,60 & - 241,37 & 236,10 & 224,54 & - & 247,65 \\
\hline
\end{tabular}

Tabela 15

Presença de EMD pela RET comparado à presença $\mathrm{EMD}$ pela $\mathrm{OCT}$

\begin{tabular}{lccccccc}
\hline & & \multicolumn{3}{c}{ EMD (OCT) } & \multirow{2}{*}{ Total } \\
\cline { 3 - 6 } & & \multicolumn{2}{c}{ Não } & \multicolumn{2}{c}{ Sim } & & \\
\cline { 2 - 6 } & $\mathbf{N}$ & $\mathbf{\%}$ & $\mathbf{N}$ & $\mathbf{\%}$ & $\mathbf{N}$ & $\mathbf{\%}$ \\
\hline \multirow{2}{*}{ EMD (RET) } & Não & $\mathbf{7 7}$ & $\mathbf{7 0 , 6 4}$ & 3 & 2,75 & 80 & 73,40 \\
& Sim & 14 & 12,84 & $\mathbf{1 5}$ & $\mathbf{1 3 , 7 6}$ & 29 & 26,60 \\
& & 91 & 83,49 & 18 & 16,51 & 109 & 100,0 \\
\hline
\end{tabular}

\section{Comparação entre a Presença de Edema Macular Diabético Endocrinologia X Oftalmologia}

Quanto à presença ou ausência de edema entre os pacientes da oftalmologia e da endocrinologia pela odds-ratio, é possível afirmar que esses têm cinco vezes mais chance de não ter EMD do que os pacientes da oftalmologia (tabela 9).

Em média, os pacientes com EMD tiveram 16 anos de diagnóstico de diabetes. Os pacientes sem EMD apresentaram menor tempo médio de diagnóstico da doença, 11 anos. A diferença entre a média do tempo do diagnóstico de diabetes entre os pacientes com EMD e os sem EMD foi estatisticamente significativa (p-valor $=0,017$ ) comprovado pela ANOVA (tabela 10).

O tempo médio de diabetes para os pacientes da endocrinologia foi de 13,3 anos, enquanto o dos pacientes da oftalmologia foi de 11,7 anos. Esse resultado não teve significância estatística (valor de $\mathrm{p}=0,498$ ). 
Tabela 16

Visão comparada com o EMD pela RET e pela OCT

\begin{tabular}{|c|c|c|c|c|}
\hline \multirow[b]{2}{*}{ Visão } & \multicolumn{2}{|c|}{$\begin{array}{c}\text { (EMD-RET) SIM X } \\
\text { EMD-OCT-NÃO }\end{array}$} & \multicolumn{2}{|c|}{$\begin{array}{c}\text { (EMD-RET) NÃO x } \\
\text { EMD-OCT-SIM }\end{array}$} \\
\hline & Endocrinologia & Oftalmologia & Endocrinologia & Oftalmologia \\
\hline $20 / 20$ & - & 2 & - & 1 \\
\hline $20 / 25$ & - & 3 & - & - \\
\hline $20 / 30$ & - & 1 & - & - \\
\hline $20 / 40$ & 3 & 1 & - & - \\
\hline $20 / 60$ & - & 1 & - & - \\
\hline $20 / 80$ & 1 & - & - & 1 \\
\hline $20 / 100$ & 1 & 1 & - & 1 \\
\hline Total & 5 & 9 & 0 & 3 \\
\hline
\end{tabular}

Tabela 17

Presença de EMD-OCT X EMD-RET por grupo de procedência

\begin{tabular}{|c|c|c|c|c|c|c|c|}
\hline \multirow[b]{3}{*}{ RET - EMD } & & \multicolumn{4}{|c|}{ Presença de edema (OCT) } & \multirow{2}{*}{\multicolumn{2}{|c|}{ Total }} \\
\hline & & \multicolumn{2}{|c|}{ Não } & \multicolumn{2}{|c|}{ Sim } & & \\
\hline & & $\mathbf{N}$ & $\%$ & $\mathbf{N}$ & $\%$ & $\mathbf{N}$ & $\%$ \\
\hline \multirow{3}{*}{ Endocrinologia } & Não & 46 & 92 & - & - & 46 & 88,46 \\
\hline & Sim & 4 & 8 & - & - & 4 & 11,54 \\
\hline & Total & 50 & 100,00 & - & - & 50 & 100,00 \\
\hline \multirow{3}{*}{ Oftalmologia } & Não & 31 & 76 & 3 & 17 & 34 & 59,65 \\
\hline & Sim & 10 & 24 & 15 & 83 & 25 & 40,35 \\
\hline & Total & 41 & 100,00 & 18 & 100,00 & 59 & 100,00 \\
\hline
\end{tabular}

Tabela 18

Comparação da medida normal média da espessura da retina (pela OCT) com a presença de EMD (pela RET) e a presença de edema (pela OCT)

\begin{tabular}{lccccc}
\hline \multirow{2}{*}{ Região } & $\begin{array}{c}\text { Média da } \\
\text { medida } \\
\end{array}$ & \multicolumn{2}{c}{$\begin{array}{c}\text { EMD RET X } \\
\text { EMD OCT NÃo }\end{array}$} & \multicolumn{2}{c}{$\begin{array}{c}\text { EMD RET NÃOX } \\
\text { EMD OCT SIM }\end{array}$} \\
\cline { 2 - 6 } & normal & Média & Valor de p & Média & Valor de p \\
\hline R1 & $\mathbf{2 1 2}$ & 207 & 0,308 & 322 & 0,235 \\
R2 & $\mathbf{2 5 5}$ & 237 & 0,141 & 301 & 0,198 \\
R3 & $\mathbf{2 6 7}$ & 264 & 0,674 & 325 & 0,135 \\
R4 & $\mathbf{2 6 0}$ & 266 & 0,539 & 320 & 0,087 \\
R5 & $\mathbf{2 5 1}$ & 259 & 0,505 & 310 & 0,167 \\
R6 & $\mathbf{2 3 9}$ & 256 & 0,056 & 288 & 0,160 \\
R7 & $\mathbf{2 4 6}$ & 244 & 0,799 & 280 & 0,141 \\
R8 & $\mathbf{2 1 0}$ & 243 & $\mathbf{0 , 0 0 1} *$ & 245 & 0,110 \\
R9 & $\mathbf{2 1 0}$ & 229 & $\mathbf{0 , 0 2 7} *$ & 241 & 0,161 \\
\hline
\end{tabular}

* p estatisticamente significativo 

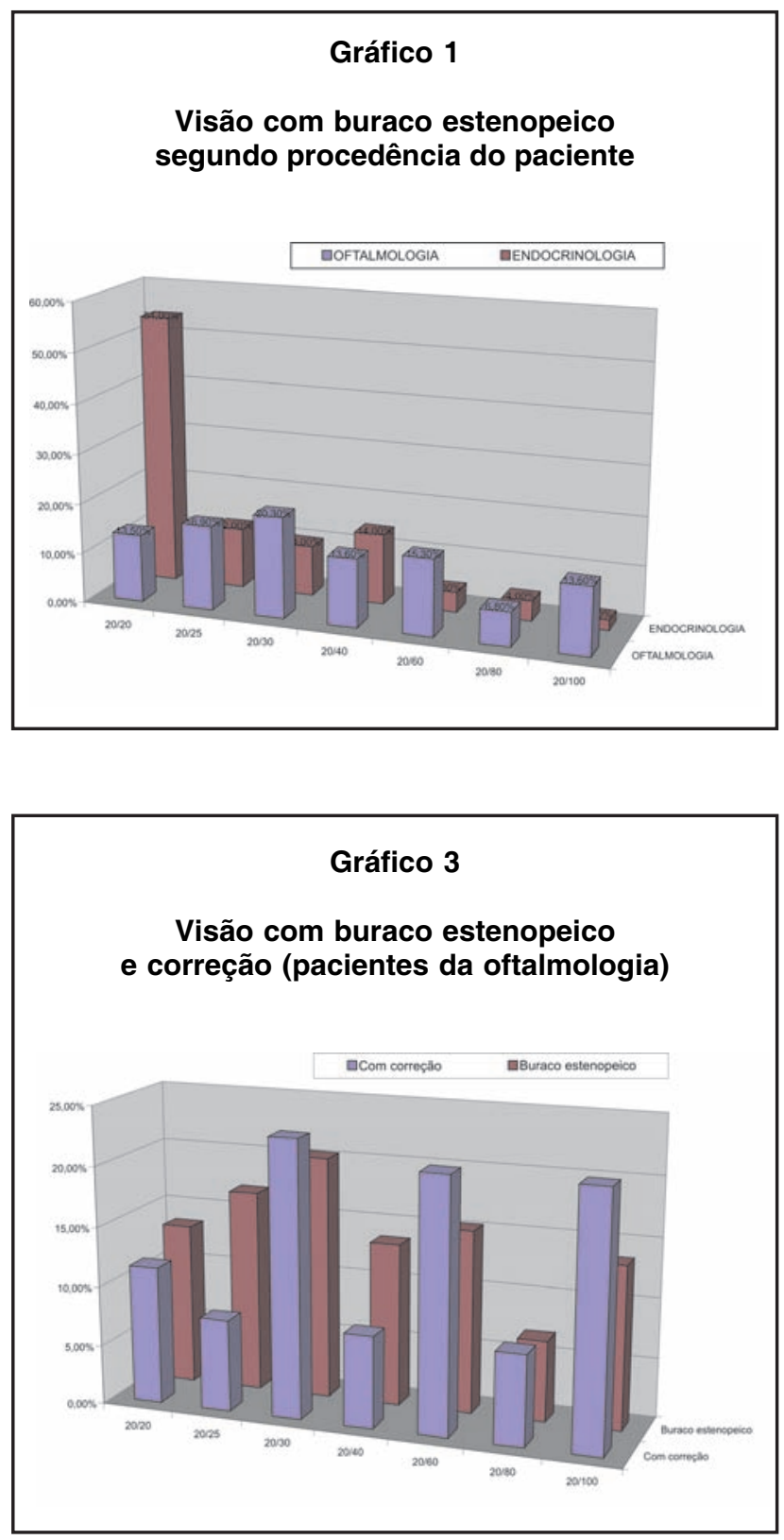

\section{Avaliação da Acuidade Visual}

\section{Acuidade visual com correção x Buraco estenopeico}

Foi aferida a AV do paciente com correção (AVCC) e com buraco estenopeico, comparando-se as medidas encontradas para cada um nos dois métodos (tabela 11).

Houve melhora significativa na $\mathrm{AV}$ dos pacientes com o buraco estenopeico. Dos 42 pacientes que não melhoraram a visão com o buraco estenopeico, 27 (47\%) eram pacientes dos serviços de endocrinologia e 31 $(53 \%)$, da oftalmologia (tabela 12$)$.

Na tabela 13, encontra-se a frequência dos olhos

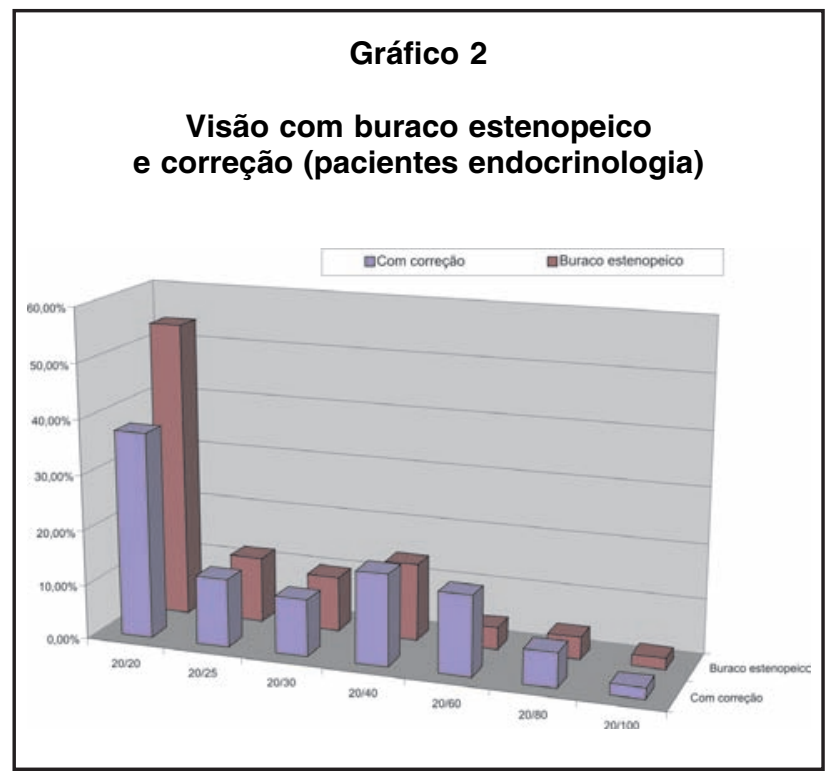

\section{Gráfico 4}

Freqüência da medida da espessura da retina na região 1 do olho segundo a acuidade visual com correção

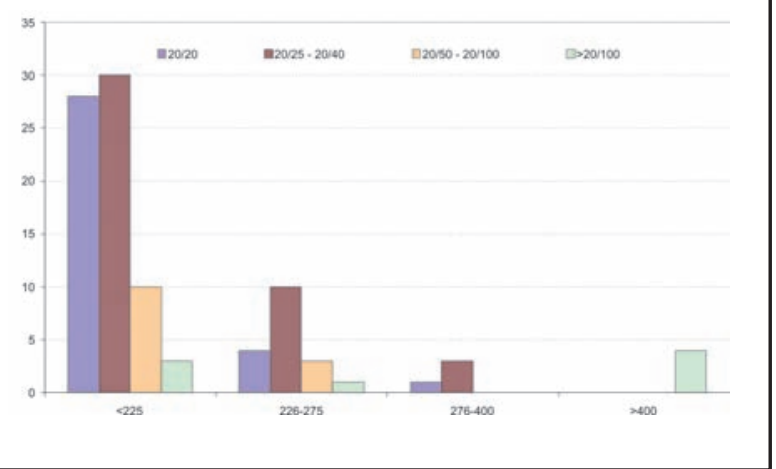

estudados, discriminados por grupo de origem e pela melhora da visão com o buraco estenopeico.

Na comparação da AV com buraco estenopeico entre os grupos, os pacientes da oftalmologia apresentaram melhor AV com buraco estenopeico. Em média, 50\% dos pacientes apresentaram visão de $20 / 40$ nos dois olhos. Aproximadamente $50 \%$ dos pacientes da endocrinologia apresentaram AV com buraco estenopeico de 20/25(gráfico 1).

Os gráficos 2 e 3 demonstram o percentual de olhos em cada categoria de AV, com correção e depois com o buraco estenopeico. 
Com correção, 37,5\% dos olhos apresentaram visão 20/20; com o buraco estenopeico, o percentual aumentou para $54 \%$.

Esse gráfico demonstra o percentual de olhos em cada categoria de AV com correção e depois com o buraco estenopeico.

Na tabela 14, encontram-se os valores médios da espessura da retina dos pacientes que não tiveram melhora da visão com o buraco estenopeico. Pela ANOVA, verificou-se que não houve diferença significativa na medida da espessura retiniana entre os pacientes que melhoraram a visão e os que não melhoraram.

Também não existe correlação estatisticamente significante entre a melhora da visão e a presença de edema (pela retinografia e OCT). Os pacientes da endocrinologia e os da oftalmologia também não têm nenhuma relação com a melhora da visão.

\section{Comparação entre o perfil do EMD pela RET pela OCT}

\section{Análise Qualitativa}

Pela RET, definiu-se se o paciente tinha ou não EMD; pela OCT, as alterações da espessura média na fenda 1 indicavam se o paciente tinha ou não edema macular em cada olho. Pela tabela 15, pode-se perceber que o resultado dos dois exames foi igual em 92 olhos, sendo 77 (70,64\%) olhos sem edema pela RET, e sem edema macular pela OCT; $15(13,76 \%)$ com edema pela RET e com edema na OCT (tabela 15).

Percebe-se que 17 (15,60\%) olhos divergiram no resultado dos dois exames. Desse total, $3(2,75 \%)$ sem edema pela RET e com edema pela OCT; os outros 14 $(12,84 \%)$, com edema pela RET, e sem edema, pela OCT.

Pelo teste de Mcnemar, foi possível verificar que, na maioria dos olhos, o diagnóstico foi semelhante nos dois exames. Mas os 17 casos que apresentaram diferença são estatisticamente significativos, sendo a OCT mais precisa nas medidas.

Quanto à presença de EMD pela RET e pela análise qualitativa da OCT, a visão desses pacientes encontrava-se assim distribuída (tabela 16):

Na relação entre presença de edema EMD (RET) e procedência dos pacientes, os resultados foram:

Na tabela 17, foram discriminados os resultados por grupo de procedência (endocrinologia ou oftalmologia). Esse último grupo apresentou maior divergência no resultado dos exames de EMD feitos com a OCT e com a RET.

\section{Análise Quantitativa pelo Software}

Pela tabela 18, pode-se verificar que não houve diferença de espessura retiniana entre a medida normal com EMD pela RET, nem sem EMD pela OCT. As regiões 8 e 9 apresentaram espessura média maior que a medida normal.

Com o gráfico 4 , demonstra-se a relação entre AVCC e espessura da retina na região 1.

\section{DısCUSSÃO}

O estudo teve como limitação o tamanho da amostra, considerado pequeno em razão da grande frequência da patologia enfocada. Do ponto de vista estatístico, um tamanho mais representativo proporcionaria um nível maior de confiança aos resultados da pesquisa, principalmente em se tratando de amostra não probabilística, selecionada por acessibilidade. No entanto, a inexistência de dados que permitissem classificar como finita a população de pacientes com essa patologia no Distrito Federal impossibilitou a ampliação da amostra.

A avaliação do tempo de diabetes é variável no Brasil e não foram encontradas, na literatura pesquisada, referências precisas quanto ao tipo de amostra utilizado, com características específicas como descrito ${ }^{(6)}$.

Diabetes é a doença multifatorial com maior número de variações de acometimento no corpo humano ${ }^{(7)}$. Essa imensa variação, por conseguinte, tem o maior número de publicações da área médica. Assim, o interesse do autor pelo assunto culminou com a elaboração de um protocolo de pesquisa que pudesse chegar o mais próximo possível da realidade do perfil de entrada do paciente em hospital público.

Chamou especial atenção as dificuldades de comparação dos dados do presente estudo com dados da literatura consultada, porque o grupo pesquisado difere dos citados nesses estudos, que traçam o perfil das alterações funcionais e, principalmente, das alterações morfológicas de pacientes com retinopatia diabética. Isso dificultou a comparação de dados obtidos na pesquisa com os dados da literatura.

\section{Tomografia de Coerência Óptica}

\section{OCT X idade}

Neste estudo, foi observado que, à medida que aumenta a idade do paciente, ocorre uma leve diminuição da espessura da retina pela OCT.

Chan et al. ${ }^{(3)}$, estudando medidas de espessura macular em indivíduos normais também por meio da OCT Stratus, não encontraram uma relação estatisticamente significante entre o aumento da idade e a diminuição da espessura retiniana. Conforme os autores, seus dados foram similares aos encontrados por Hee et al. ${ }^{(8)}$ e por 
Sanchez-Tocino et al. ${ }^{(9)}$, apesar das suspeitas de que a retina pode diminuir de espessura com o passar da idade.

Neste trabalho, através do teste de Pearson, observou-se uma correlação negativa com significância estatística nas regiões 4,5,6,7,8 e 9. A diferença entre os estudos indicados acima pode ter ocorrido em razão de a amostra ser de pacientes diabéticos e a amostra dos outros estudos ter sido composta por pacientes não diabéticos.

\section{Tempo de Diabetes x EMD}

Os pacientes com edema não apresentaram maior tempo de diabetes que os pacientes sem edema. Não houve correlação significativa entre o tempo de diabetes e o aumento da espessura retiniana.

Pela ANOVA, não houve diferença estatisticamente significativa entre a presença de edema e o tempo de diabetes nem pela OCT, nem pela RET. Contudo, segundo Klein et al. ${ }^{(10)}$, o WESDR demonstrou haver um risco de desenvolvimento de EMD nas seguintes situações e índices de probabilidades: 20,1\% em pacientes que foram acometidos de diabetes ainda jovens; $25,4 \%$ em diabéticos, cujo início ocorreu em idade adulta e utilizam insulina; $13,9 \%$ no grupo de pacientes que adquiriram a doença na idade adulta e não utilizam insulina.

\section{Análise Quantitativa da OCT}

Nos pacientes com edema, os valores médios da espessura retiniana por regiões pela OCT foram maiores que a medida da retina dos pacientes sem edema, confirmando o esperado pela avaliação clínica.

$\mathrm{Na}$ tabela 4, foi comparada a espessura retiniana média por regiões entre o grupo da endocrinologia e o grupo da oftalmologia, sendo observada espessura média maior no segundo grupo, confirmando os achados de que o grupo (oftalmologia) teve uma incidência maior de EMD.

Em relação às espessuras (tabela 5), quando comparadas aos valores normais publicados em 1996 por Chan et al. ${ }^{(11)}$, verifica-se que: nos pacientes sem edema, as medidas das regiões 1,2 , e 3 foram menores do que as da região normal; nas regiões 6,8 , e 9 , a espessura foi maior do que o normal. Contudo, as medidas 1, 3, 6 e 9 encontravam-se dentro do intervalo de confiança. Somente as regiões 2 e 8 apresentaram $13 \mu \mathrm{m}$ a menos e $12 \mu \mathrm{m}$ a mais, respectivamente.

Diante disso, pode-se afirmar que os pacientes sem edema ou normais tinham sua espessura retiniada comparáveis à dos pacientes sem qualquer patologia associada. Já nos pacientes com edema, todas as regiões esta- vam bem acima dos valores considerados normais, também confirmando os resultados de Chan et al ${ }^{(11)}$.

\section{Análise Qualitativa da OCT}

Chan et al. ${ }^{(3)}$ quando estudaram a espessura macular de indivíduos normais através da OCT Stratus, utilizaram protocolo de seis cortes consecutivos, passando pela fóvea. As imagens foram analisadas pelo software, que utiliza a técnica de detecção das bordas mais reflectivas, como se fosse a interface vítreorretiniana e a superfície anterior do epitélio pigmentar da retina. Em complementação à análise quantitativa do programa, foi feita uma medida manual da área central de cada corte, para obter a média da espessura central (fenda 1). A média da medida central pela avaliação manual foi de $170 \pm 18 \mu \mathrm{m}$, aproximadamente $12 \mu \mathrm{m}$ menos do que a análise automática. Esses dados demonstram que a aferição automática do software, mesmo em pacientes com meios completamente transparentes (normais), pode ser diferente da análise manual, o que justificou a inclusão de nossa avaliação denomidada de qualitativa.

Os dados da tabela 7, representativos da comparação dos valores dos olhos dos pacientes classificados com e sem edema, pela avaliação da fenda central na análise qualitativa da OCT, demonstraram que foi significativa a diferença entre os resultados, validando os achados obtidos nessa análise.

\section{Retinografia}

\section{Tempo de Diabetes X EMD}

O tempo médio de diabetes foi de 3,7 anos, maior nos pacientes que apresentaram edema pela RET na região 9 do olho. Contudo, de forma geral, não apresentou diferença estatisticamente significativa (Tabela 8).

\section{EMD Grupo Endocrinologia X Oftalmologia}

Diante dos resultados da tabela 9 , reitera-se que os pacientes da oftalmologia têm mais chance de apresentar edema do que os pacientes da endocrinologia. Esse resultado confirma dados da literatura, segundo os quais um paciente melhor controlado tem menor índice de retinopatia e, consequentemente, menor indicação de tratamento. No estudo de Wisconsin, publicado por Klein et al. ${ }^{(12)}$, demonstrou-se que, após 10 anos de duração do diabetes, a severidade da retinopatia foi relacionada a maior duração de diabetes, hemoglobina glicada elevada, proteinúria e alta da pressão arterial diastólica.

Comparando os resultados da RET (tabela 12) entre os dois grupos, através do teste Qui-quadrado e do teste razão de chance (odds-ratio), foi possível con- 
firmar, com significância estatística, que os pacientes da oftalmologia têm mais chance de apresentar edema do que os pacientes da endocrinologia. Esses resultados podem ser explicados pelo fato de, na amostra pesquisada, os pacientes, selecionados aleatoriamente do setor hospitalar de oftalmologia entre os que aguardavam por exames, encontrarem-se em condições menos favoráveis do que a do grupo de pacientes da endocrinologia. Contudo, o tempo médio de diabetes nesse grupo era maior do que o do grupo da oftalmologia, contrariando o estudo de Wisconsin ${ }^{(12)} \mathrm{com}$ relação à duração do diabetes, sugerindo que realmente esse grupo deveria estar melhor controlado sistemicamente.

$\mathrm{Na}$ análise pela razão de chance (odds-ratio), o grupo da oftalmologia apresentou cinco vezes mais chance de ter EMD do que o grupo da endocrinologia, demonstrando, outra vez, que o paciente com melhor controle sistêmico tem menor risco de desenvolver edema macular

Confirmam-se, assim, os dados do estudo DCCT ${ }^{(13)}$, que já em 1993 demonstraram que a redução de 10\% no nível da hemoglobina glicada reduz o risco do início da retinopatia diabética, inibe a progressão do EMD e também a necessidade de tratamento por laser.

\section{Acuidade visual com correção $X$ Buraco estenopeico}

Munhoz et al. ${ }^{(14)}$ estudaram a cegueira, a baixa visual e o problema do erro de refração em uma população de americanos de origem mexicana, demonstrando que dois terços dessa população poderiam melhorar sua visão com a prescrição de novos óculos. As causas apontadas para a solução foram: limitações em procurar médicos, devido a dificuldades com o idioma; dificuldade financeira e falta de informação sobre os serviços públicos disponíveis. Segundo esses autores, seus dados concordam com o resultado de estudos prévios, como os de Tielsch et al. ${ }^{(15)}$, Rubin et al. ${ }^{(16)}$ e Attebo et al. ${ }^{(17)}$

Nesta pesquisa, a AV dos pacientes envolvidos melhorou em $47 \%$ com o uso de buraco estenopeico, indicando que eles poderiam se beneficiar com a simples troca dos óculos. Analisando a melhora da visão por grupo de origem, o da oftalmologia teve discreto percentual de melhora a mais, porém sem diferença estatisticamente significativa. A dificuldade financeira apontada nos estudos anteriores parece ser a causa em comum.

Na tabela 13 , observa-se que 18 olhos da endocrinologia e 6 da oftalmologia apresentavam AV corrigida de 20/20 (100\% de visão), indicando que ape- nas em 22\% (24/109) dos olhos apresentavam 100\% da visão corrigida sem EMD, não necessitando melhorar refração.

Nove olhos dos pacientes da endocrinologia e dois da oftalmologia melhoraram a visão com buraco estenopeico para 20/20, indicando que 10\% (11/109) dos olhos poderiam estar com $100 \%$ com a mudança dos óculos.

Quatorze olhos dos pacientes da endocrinologia e 26 da oftalmologia, 36\% (40/109) poderiam se beneficiar com novos óculos, mas sem atingir $100 \%$ da visão. No estudo de Munhoz et al. ${ }^{(14)}$, dos 4.255 indivíduos examinados, $73 \%$ melhoraram sua acuidade visual para mais de 20/40, sendo que, nessa população, havia 18,6\% que sabiam ser diabéticos (885/4.255). Se se calcular o percentual de $73 \%$ sobre a população de diabéticos do estudo, verifica-se que o percentual de melhora dessa população é de $28,4 \%$.

Pelos resultados das tabelas 14 e 16, observa-se que não houve correlação significativa entre a melhora da visão com buraco estenopeico e a presença de edema, nem pela OCT nem pela RET, confirmando o dado de que a melhora da visão poderia ser obtida com a atualização dos óculos.

O gráfico 4 mostra que mais de $50 \%$ dos olhos com AV melhor do que 20/40 se encontrava com a espessura retiniana menor do que 225 micra. Esse resultado não pode ser comparado com o do estudo de $\mathrm{DRCR}^{(18)}$, por ter sido utilizada escala de Snellen, em vez de os optótipos do ETDRS. Contudo, ficou demonstrado que os pacientes com espessura retiniana próxima do normal apresentavam acuidade visual melhor.

\section{EMD pela RET X EMD pela OCT}

Foi observada diferença estatisticamente significativa entre as espessuras por grupo de origem entre os pacientes da endocrinologia e da oftalmologia pela análise do software (tabela 4). Em média, os pacientes do ambulatório da oftalmologia apresentaram espessamento maior do que o do grupo da endocrinologia, apesar de esse último ter média de tempo de diabetes maior que o primeiro.

Pela análise qualitativa (manual, sem a utilização do software), vê-se que $100 \%$ dos pacientes com edema eram do grupo da oftalmologia (tabela 8). A provável explicação para esse resultado pode ser o fato de o grupo da endocrinologia estar mais compensado metabolicamente do que o da oftalmologia.

Esses dados estão de acordo com os do estudo do DCCT $^{(13)}$, segundo o qual o controle intensivo do diabetes efetivamente retarda o início da retinopatia diabética ou diminui sua progressão. 
Na tabela 15 , vê-se que, pela OCT, $13 \%$ dos pacientes sem EMD tiveram EMD pela RET; 3\% tinham edema pela OCT e não apresentavam EMD pela RET. A explicação para esses resultados é que o diagnóstico pela avaliação qualitativa foi feito através da fenda central, e os pacientes com EMD pela RET apresentavam um espessamento que ameaçava a área central; nos 3\% com edema, o espessamento pode não ter sido detectada pela avaliação retinográfica.

Nos dados dessa Tabela, compara-se a espessura retiniana média normal por regiões com a EMD pela RET, demonstrando que as regiões 8 e 9 estavam mais espessadas que a média normal, com significância estatística. Isso confirma a explicação dada na tabela 14, na qual não se verificou espessamento central, embora houvesse ameaça macular nas regiões inferior e temporal.

Em 2007, o DRCR ${ }^{(18)}$ comparou a região central foveal pela OCT com a AV antes e depois de fotocoagulação por laser, na tentativa de relacionar a $\mathrm{AV}$ com a espessura retiniana, para que as medidas encontradas servissem de padrão objetivo nos estudos multicêntricos. Nesse trabalho, havia uma substancial variação na $\mathrm{AV}$ com as medidas de espessura foveais. Muitos olhos com mácula espessada apresentavam excelente AV, e outros apresentavam espessura macular normal, com baixa AV.

Nesta pesquisa, foi feita uma avaliação morfofuncional de pacientes diabéticos, determinando o perfil da AV e sua melhora com nova correção de óculos, relacionado com a espessura retiniana avaliada por dois métodos importantes e viáveis nos hospitais públicos. Foi observada que uma grande parte dos pacientes encontrava-se bem da retinopatia e poderia se beneficiar com um simples exame de refração. Sendo assim, este estudo acrescentou à literatura dados que deverão ser utilizados para pesquisas de maior amplitude, otimizando a prevenção e a qualidade de vida de pacientes diabéticos, que representam um problema de saúde pública em qualquer lugar do mundo.

\section{Conclusão}

O perfil de espessura retiniana pela OCT na amostra estudada demonstra que quanto maior a idade menor a espessura retiniana e que não houve influência do tempo de diabetes na espessura retiniana.

O perfil do EMD verificado pela RET demonstrou que também não houve correlação estatisticamente significativa entre o tempo de diabetes e a presença de EMD.

\section{Abstract}

Objective: To demonstrate the morpho-functional profile of the evaluation of patients with diabetic retinopathy without severe loss of visual acuity in a public hospital of reference in endocrinology, determining in this sample, the relation between the time of diabetes, age and visual acuity with the retinal thickness measured by the optical Coherence tomography (OCT) and fundus picture (FP). Methods: Prospective, linear study was carried through, in transversal cut of 61 consecutive patients with diabetic retinopathy registered in the $H R T$, and refered from the services of ophthalmology and endocrinology. Patients had been submitted to a complete ophthalmic evaluation including clinical history, visual acuity with correction and pin hole. The patients who had presented diabetic retinopathy, with transparent media, without previous surgery, nor previous Laser photocoagulation and with visual acuity better than 20/100 at Snelen scale, had been included in the study. After the elimination of the patients who had not obeyed the inclusion/exclusion criteria 109 eyes of 55 patients then had been submitted the OCT and FP for evaluation of the presence or absence of edema by the FP and of the quantitative evaluation (measured of the retinal thickness of the 9 regions of the pelo Early Tratment Diabetic Retinophaty Study (ETDRS), and of the qualitative evaluation (presence or absence of retinal edema for the central slit, number 1). Results: The average time of diabetes was of 12 years, varying of 23 to 86 years old. $51 \%$ were female, and $49 \%$ male. The OCT demonstrated discrete reduction of the retinal thickness with elapsing of the age. The patients of the ophthalmology had greaters values of retinal thickness of what of the group of the endocrinology. The evaluation of the visual acuity improved with pin hole in 47\% (51/109). The endocrinology group were $45 \%$ (23/50) of the eyes and the ophthalmology group were 55\% (28/59). Only 22\% (24/109) of the eyes presented corrected visual acuity of 20/20 with correction and without Diabetic Macular Edema (DME). In 83\% (91/109) of the cases it had agreement and in 17\% (18/109 eyes) it had discord in relation the presencelabsence of DME in the profile of the morphologic evaluation carried through by the optic coherence tomography and the fundus picture, in the eyes of the amostral group. Conclusion: It did not have statistical significant correlation between the retinal thickness and the time of diabetes.

Keywords: Diabetic retinopathy; Optical coherence tomography; Macular edema; Ophthalmological diagnostic techniques/utilization; Retina/ radiography; Visual acuity 


\section{RefERÊNCIAS}

1. Bailey CC, Sparrow JM, Grey RH, Cheng H. The National Diabetic Retinopathy Laser Treatment Audit. III. Clinical outcomes. Eye. 1999;13(Pt 2):151-9.

2. Campbell RJ, Coupland SG, Buhrmann RR, Kertes PJ. Optimal optical coherence tomography-based measures in the diagnosis of clinically significant macular edema: retinal volume vs foveal thckness. Arch Ophthalmol. 2007; 125 (5):619-23.

3. Chan A, Duker JS, Ko TH, Fujimoto JG, Schuman JS. Normal macular thickness measurements in healthy eyes using Stratus optical coherence tomography. Arch Ophthalmol. 2006;124 (2):193-8.

4. Early Treatment Diabetic Retinopathy Study design and baseline patient characteristics. ETDRS report number 7 . Ophthalmology. 1991;98 (5 Suppl):741-56.

5. Photocoagulation for diabetic macular edema. Early treatment Diabetic Retinopathy Study report number 1. Early Treatment Diabetic Retinopathy Study research group. Arch Ophthalmol. 1985;103:1796-806.

6. Abujamra S, Ávila M, Barsante C, Farah ME, Gonçalves JO, Lavinsky J, et al. Retina e vítreo: clínica e cirurgia. São Paulo: Rocca; 2000.

7. Nehemy MB. Retinopatia diabética. Arq Bras Oftalmol. 2008;71(3). [citado 2008 Out 10]. Disponível em: http:// www.abonet.com.br/abo/ revisao3.htm

8. Hee MR, Puliafito CA, Wong C, Duker JS, Reichel E, Rutledge B, et al. Quantitative assessment of macular edema with optical coherence tomography. Arch Ophthamol. 1995;113 (8):1019-29.

9. Sanchez-Tocino H, Alvarez-Vidal A, Maldonado MJ, MorenoMontañés J, García-Layana A. Retinal thickness study with optical coherence tomography in patients with diabetes. Invest Ophthalmol Vis Sci. 2002, 43(5): 1588- 94

10. Klein R, Meuer SM, Moss SE, Klein BE. Retinal microaneurysm counts and 10-year progression of diabetic retinopathy. Arch Ophthalmol. 1995;113 (11):1386-91.

11. Chan A, Duker JS, Schuman JS, Fujimoto JG. Stage 0 macular holes: observations by optical coherence tomography. Ophthalmology. 1996;111(11):2027-32.
12. Klein R, Klein BE, Moss SE, Davis MD, DeMets DL. The Wisconsin epidemiologic study of diabetic retinopathy. II. Prevalence and risk of diabetic retinopathy when age at diagnosis is less than 30 years. Arch Ophthalmol. 1984;102(4):520-6.

13. DCCT. The Diabetes Control and Complications Trial Research Group. The effect of intensive treatment of diabetes on the development and progression of long-term complications in insulin-dependent diabetes mellitus. N Engl J Med. 1993;329 (14):977-86.

14. Muñoz B, West SK, Rodriguez J, Sanchez R, Broman AT, Snyder $\mathrm{R}$, Klein R. Blindness, visual impairment and the problem of uncorrected refractive error in a Mexican-American population: Proyecto VER. Invest Ophthalmol Vis Sci. 2002;43 (3):608-14

15. Tielsch, JM, Sommer, A, Witt, K, Katz, J, Royall, RM. Blindness and visual impairment in an American urban population. Arch Ophthalmology. 108(2):286-90.

16. Rubin GS, West SK, Muñoz B, Bandeen-Roche K, Zeger S, Schein O, Fried LP. A comprehensive assessment of visual impairment in a population of older Americans: the SEE study. Invest Ophthalmol Vis Sci. 1997;38(3):557-68.

17. Attebo K, Mitchell P, Smith W. Visual acuity and the causes of visual loss in Australia: the Blue Mountains Eye Study. Ophthalmology. 1996; 103(3):357-64.

18. Diabetic Retinopathy Clinical Research Network. Comparison of the modified early treatment diabetic retinopathy study and mild macular grid laser photocoagulation strategies for diabetic macular edema. Arch Ophthalmol. 2007; 125(4):469-80.

\section{Endereço para correspondência \\ ICOB - Instituto de Cirurgia Ocular de Brasília \\ SGAS 915 Ed.Office Center Bloco B - $2^{\circ}$ andar CEP 70390-150 - Brasília (DF), Brasil.}

Tel: 6133452424

E-mail: dr.alipio@icob.med.br 